\title{
Hipertensión arterial resistente
}

\author{
Carlos A. Feldstein
}

\section{Resistant hypertension}

Resistant hypertension, defined as a persistent blood pressure over $140 / 90 \mathrm{mmHg}$ despite the use of three antihypertensive drugs including a diuretic, is unusual. The diagnosis requires ruling out initially pseudoresistance and a lack of compliance with treatment. Ambulatory blood pressure recording allow the recognition of white coat hypertension. When there is a clinical or laboratory suspicion, secondary causes of hypertension should be discarded. Excessive salt intake, the presence of concomitant diseases such as diabetes mellitus, chronic renal disease, obesity, and psychiatric conditions such as panic attacks, anxiety and depression, should also be sought. The presence of target organ damage requires a more aggressive treatment of hypertension. Recent clinical studies indicate that the administration of aldosterone antagonists as a fourth therapeutic line provides significant additional blood pressure reduction, when added to previous antihypertensive regimens in subjects with resistant hypertension. The possible blood pressure lowering effects of prolonged electrical activation of carotid baroreceptors is under investigation (Rev Méd Chile 2008; 136: 528-38).

(Key w ords: Aldosterone antagonists; Hypertension; Pressoreceptors)

Recibido el 2 de mayo, 2007. Aceptado el 23 de julio, 2007.

$\mathrm{L}$ a hipertensión arterial (HTA) resistente es una condición que involucra alto riesgo de daño de órganos blanco, con significativo aumento de morbimortalidad. El $7^{\text {th }}$ Report of the Joint National Committee on the Detection, Evaluation and Treatment of High Blood Pressure en $2003^{1}$ estableció criterios, incorporados en 2007 por la European Society of Hypertension/European Society of Cardiology (ESH/ESC) ${ }^{2}$ que definen la HTA resistente al tratamiento, como aquélla con niveles de presión arterial (PA) iguales o superiores a 140/ $90 \mathrm{mmHg}$, a pesar de cumplir el tratamiento con un régimen apropiado de 3 fármacos antihipertensivos, incluyendo un diurético. En pacientes con

Correspondencia a: Dr. Carlos A. Feldstein. Director del Programa Hipertensión Arterial, Hospital de Clínicas José de San Martín, UBA. Av. Córdoba 2351, 7o Piso. Buenos Aires 1120, Argentina. E mail: carlfel@yahoo.com comorbilidades como diabetes mellitus o enfermedad renal crónica (creatinina $>1,5 \mathrm{mg} / \mathrm{dl}$ o proteinuria $>300 \mathrm{mg} / 24 \mathrm{~h}$ ), la HTA se considera resistente con niveles iguales o superiores a 130/ $80 \mathrm{mmHg}$. Los grandes ensayos clínicos han mostrado que las elevaciones de la PA diastólica resultan más fáciles de controlar que las de la PA sistólica. Así, en el Antihypertensive and LipidLowering Treatment to Prevent Heart Attack Trial (ALLHAT), después de 5 años de seguimiento, 92\% de los pacientes tuvo niveles de PA diastólica $<90 \mathrm{mmHg}$ mientras que sólo en $67 \%$ la PA sistólica fue $<140 \mathrm{mmHg}^{3}$.

\section{Prevalencia}

Aun cuando los datos disponibles en la literatura son escasos y muestran disparidades entre diversos estudios, la HTA resistente es más frecuente en 
pacientes mayores de 60 años de edad y en aquéllos con daño de órganos blanco ${ }^{4}$. Su prevalencia en centros especializados en HTA es más elevada (11\%-13\%) ${ }^{5,6}$ que en la población total de hipertensos $(2 \%-5 \%)^{7}$. Los estudios de seguimiento en que el protocolo impone el tipo y la dosis de drogas a utilizar permiten estimar mejor la prevalencia. Así, en el ALLHAT ${ }^{8}$ que incluyó más de 33.000 pacientes con un promedio de seguimiento de aproximadamente 5 años, $15 \%$ de la cohorte podría clasificarse como HTA resistente?. En el estudio Controlled Onset Verapamil Investigation of Cardiovascular End Points (CONVINCE) ${ }^{10}$ efectuado en hipertensos de más de 55 años con al menos 1 factor de riesgo cardiovascular además de la HTA, 18\% estaba tratado con 3 o más agentes antihipertensivos, cumpliendo los criterios de HTA resistente. La tendencia declinante en la prevalencia de la HTA resistente se puede atribuir a la evolución de las técnicas de diagnóstico y al empleo combinado de las modernas familias de fármacos antihipertensivos.

\section{DiAgNóstico}

La clara separación entre quienes realmente tienen resistencia al tratamiento y los que presentan pseudorresistencia permite reducir en estos últimos la indicación de estudios complementarios y ajustes terapéuticos innecesarios. La pseudorresistencia incluye a la HTA de delantal blanco y la pseudohipertensión. La HTA de delantal blanco en que las cifras elevadas de PA sólo se comprueban en consultorio, tiene una prevalencia de aproximadamente $20 \%{ }^{11}$. Debe sospecharse en pacientes con cifras normales de PA en domicilio, que presentan síntomas secundarios a hipotensión arterial al aumentar las dosis de antihipertensivos, y en aquellos hipertensos de larga evolución sin evidencias de daño de órgano blanco. Garg y cols ${ }^{12}$, en un tercio de sus pacientes con aparente HTA resistente, comprobaron niveles promedios de PA inferiores a 130/85 mm Hg en el monitoreo ambulatorio de PA (MAPA) de $24 \mathrm{~h}$. Muxfeldt y cols ${ }^{13}$ sostuvieron que el MAPA de $24 \mathrm{~h}$ es el único método no invasivo que permite diferenciar la HTA de delantal blanco de la verdadera HTA resistente.

La pseudohipertensión, por otro lado, es una condición en que la medición indirecta de la PA con esfigmomanómetro es artificialmente más elevada en comparación con la presión intraarterial. Ocurre más comúnmente en pacientes con arteriosclerosis avanzada (ancianos, falla renal crónica progresiva y en diabéticos). Es el resultado de la fibrosis o calcificación arterial, que disminuyen marcadamente su colapsabilidad, de modo que se palpan distalmente los latidos de las arterias humeral y radial, en niveles más elevados que los correspondientes a la PA sistólica intraarterial (signo de Osler) ${ }^{14}$. El diagnóstico de pseudohipertensión sólo puede descartarse por cateterismo arterial. Se sospecha si en un anciano las cifras elevadas de PA sistólica no coexisten con daño de órganos blanco, si el tratamiento antihipertensivo induce síntomas de hipoperfusión, sin reducción de la PA y si se comprueba radiográficamente la calcificación de la arteria humeral ${ }^{15}$. La maniobra de Osler no es confiable para descartar la pseudohipertensión, pudiendo ser positiva aun en ausencia de ésta y en hipertensos con manifestaciones de daño de órganos nobles ${ }^{16}$.

La falta de adherencia al tratamiento es una causa frecuente de control inadecuado de la $\mathrm{PA}^{17}$. Los ancianos constituyen la cohorte con mayor prevalencia de HTA resistente, lo que puede atribuirse a las instrucciones inadecuadas suministradas por el médico, a las dificultades cognoscitivas para la comprensión de prescripciones de varios fármacos, a efectos colaterales de los antihipertensivos y a razones económicas ${ }^{18}$.

Patogenia de la HTA resistente. Las causas más frecuentes de la verdadera HTA resistente se hallan en la Tabla 1.

Relacionadas con las drogas: la terapia subóptima parece ser la causa más frecuente de HTA resistente, en especial la prescripción de dosis insuficientes de diuréticos ${ }^{18}$. Los responsables pueden ser los propios médicos, cuando no intensifican la terapia antihipertensiva ante la ausencia de una adecuada respuesta terapéutica ${ }^{19}$. Así, Berlowitz y col${ }^{20}$, en aproximadamente $40 \%$ de los ancianos hipertensos seguidos durante 2 años, observaron niveles iguales o superiores a 160/90 mmHg, a pesar de que efectuaron un promedio de 6 consultas médicas anuales vinculadas al manejo de la HTA. Las interacciones medicamentosas pueden reducir el efecto de los antihipertensivos al alterar su absorción, metabo- 


\section{Tabla 1. C ausas de hipertensión arterial resistente}

1) Relacionadas con las drogas

Dosis insuficientes

Combinaciones inapropiadas

Rápida inactivación (hidralazina)

Interacción con otras drogas: corticoides, antiinflamatorios no esteroides (AINES), descongestivos nasales, simpaticomiméticos, antidepresivos, supresores del apetito, anticonceptivos orales, ciclosporina, tacrolimus, eritropoyetina, cocaína, hierbas (ginseng, yohimbina), esteroides anabólicos

2) Condiciones asociadas

Sobrepeso-obesidad

Tabaquismo

Excesiva ingesta de alcohol

Insuficiencia renal

Resistencia a la insulina

Dolor crónico

Trastornos de ansiedad-ataque de pánico

Intensa vasoconstricción (arteritis, fenómeno de Raynaud)

3) Sobrecarga de volumen

Inadecuada terapia con diuréticos

Exceso de ingesta de sal

Retención de fluidos secundaria a la disminución de la PA con antihipertensivos

Daño renal progresivo (nefroesclerosis)

4) Hipertensión secundaria

lismo o excreción (Tabla 2). En una evaluación retrospectiva de las causas de HTA resistente, Garg y col ${ }^{12}$ señalaron en $58 \%$ su vinculación con las drogas utilizadas, en 9\% con causas psicológicas, y en 5\% con HTA secundaria. El empleo habitual de ciertas medicaciones por interacciones, puede causar resistencia al tratamiento antihipertensivo (Tabla 2). El efecto de los calcioantagonistas es menos afectado que el de los inhibidores de la enzima de conversión de angiotensina (IECAs) y los diuréticos por la administración concomitante de antiinflamatorios no esteroidales (AINEs) ${ }^{21}$. La aspirina en dosis de 80 $\mathrm{mg} /$ día no interfiere con el efecto de las drogas antihipertensivas ${ }^{22}$.

Condiciones asociadas: se ha analizado la relación entre trastornos psicológicos e HTA resistente. Davies y cols ${ }^{23}$, en un estudio caso-control, no hallaron diferencias en la prevalencia de ataques de pánico, ansiedad y depresión entre pacientes con HTA resistente e hipertensos respondedores. Sin embargo, comprobaron que las crisis de pánico tuvieron una prevalencia remarcablemente elevada en ambos grupos (33\% y 39\%, respectivamente). Kaplan ${ }^{24}$ halló hiperventilación inducida por ansiedad en 96 de 300 pacientes evaluados. En otro estudio, Davies y cols ${ }^{25}$ señalaron que la intolerancia medicamentosa por efectos adversos inespecíficos en hipertensos se relacionó con ataques de pánico y otras manifestaciones de morbilidad psiquiátrica. Esos resultados indican la conveniencia de evaluar los posibles factores psicológicos que pudieran tener un papel en la resistencia al tratamiento de la HTA.

Entre las comorbilidades, se destaca la incidencia significativa de HTA resistente en los diabéticos ${ }^{26}$.

En hipertensos de escasos recursos económicos, la HTA severa pobremente controlada se relaciona con la falta de controles médicos e incumplimiento del tratamiento farmacológico.

El consumo excesivo habitual de bebidas alcohólicas se relaciona fuertemente con la incidencia de HTA $^{27,28}$. Cuando es mayor de 2 "tragos" estándar diarios (un "trago" equivale a 14 g de 
Tabla 2. Interacciones entre drogas que pueden causar H TA resistente al tratamiento

\begin{tabular}{|c|c|c|}
\hline Droga antihipertensiva & Droga que interactúa & Mecanismo de interacción \\
\hline Hidroclorotiazida & Colestiramina & Disminuyen la absorción \\
\hline Furosemida & Sucralfato & \\
\hline Propranolol & Rifampicina & $\begin{array}{l}\text { Incrementa el metabolismo } \\
\text { hepático }\end{array}$ \\
\hline $\begin{array}{l}\text { Inhibidores de enzima convertidora, } \\
\text { bloqueadores del receptor de } \\
\text { angiotensina, } § \text { bloqueantes, } \\
\text { Hidralazina, diuréticos }\end{array}$ & AINEs & $\begin{array}{l}\text { Disminuyen las prostaglandinas } \\
\text { vasodilatadores renales, y } \\
\text { aumentan la retención de sodio }\end{array}$ \\
\hline Alfa-metildopa, clonidina & Naloxona & $\begin{array}{l}\text { Disminuye la inhibición del } \\
\text { sistema nervioso central y } \\
\text { aumenta la actividad simpática }\end{array}$ \\
\hline Todos las drogas antihipertensivas & $\begin{array}{l}\text { Cocaína, } \\
\text { antidepresivos tricíclicos }\end{array}$ & $\begin{array}{l}\text { Disminuyen la recaptación de } \\
\text { noradrenalina a nivel } \\
\text { presináptico }\end{array}$ \\
\hline Todos las drogas antihipertensivas & $\begin{array}{l}\text { Fenilpropanolamina y otros } \\
\text { simpaticomiméticos }\end{array}$ & $\begin{array}{l}\text { Estimulación directa del } \\
\text { receptor adrenérgico }\end{array}$ \\
\hline
\end{tabular}

etanol, presentes en 12 onzas o $350 \mathrm{ml}$ de cerveza, 5 onzas o $145 \mathrm{ml}$ de vino y 1,5 onzas o $45 \mathrm{ml} \mathrm{de}$ bebidas espirituosas destiladas al 40\%), más alta es la PA y su resistencia al tratamiento ${ }^{29}$. La PA disminuye a los pocos días de haber reducido significativamente o suprimido la ingesta de bebidas alcohólicas, habiéndose sugerido que el etanol interfiere con los efectos de los antihipertensivos ${ }^{30}$. Al fumar cigarrillos se elevan en forma aguda la PA y la frecuencia cardíaca y aunque no es causa de HTA, reduce el efecto de las drogas antihipertensivas. Los niveles séricos de propranolol disminuyen aproximadamente $37 \%$ en fumadores, posiblemente por aumento de su metabolismo, al estimularse el citocromo $\mathrm{P} 450^{30}$.

Sobrecarga de volumen: es frecuente en pacientes con HTA resistente. La sensibilidad al sodio, definida como aumento de la PA en respuesta a un consumo más elevado de sal que con la dieta basal, puede ocurrir en normotensos e hipertensos ${ }^{31}$. Los riñones tienen un papel fundamental en la patogénesis de la HTA esencial $^{32}$. Estudios seminales de Dahl y Heine ${ }^{33}$ y otros $^{34}$ demostraron que la HTA puede ser transferida por trasplante renal de una rata genéticamente hipertensa a ratas normotensas. En forma similar, Curtis y cols ${ }^{35}$ comprobaron que la HTA en pacientes de raza negra con nefropatía hiper- tensiva podía curarse por trasplante renal desde donantes normotensos. Entre los mecanismos adicionales para la retención de sodio se hallan la reducción congénita en el número de nefronas, la disminución del flujo medular renal y una subrepticia injuria renal adquirida por isquemia o inflamación del intersticio ${ }^{36}$. La exagerada ingesta de sodio, la insuficiencia renal subyacente y la retención reactiva de sodio que ocurre con el uso de algunas clases de antihipertensivos no diuréticos, como los beta-bloqueantes, los bloqueantes alfa- y beta-adrenérgicos y los vasodilatadores directos, aumenta el volumen plasmático y disminuye o anula el efecto reductor de la PA. Los calcioantagonistas, IECAs y los bloqueantes de receptores de angiotensina II (BRAs) no producen retención hídrica. La ingesta excesiva de sal disminuye el balance negativo de sodio y agua, y contribuye al desarrollo de HTA resistente. Una forma relativamente fácil de comprobar el exceso de consumo de sal es por una natriuria de $24 \mathrm{~h}$ superior a 100-120 mEq. La sensibilidad a la sal está aumentada en mayores de 60 años de edad, falla renal crónica, diabetes mellitus, obesos y negros. Más de 40\% de los pacientes con HTA resistente son obesos ${ }^{37}$.

HTA secundaria: los indicios clínicos que sugieren una causa secundaria de la HTA se hallan 
en la Tabla 3. Su prevalencia se estima entre 5\%$10 \%$ del total de hipertensos. Debe sospecharse en todo paciente con HTA catalogada como esencial en quien en forma rápida se instala una falta de efectividad del régimen antihipertensivo, con el que previamente se controlaban adecuadamente las cifras tensionales ${ }^{38,39}$. Anderson y cols ${ }^{40}$ evaluaron 4.000 pacientes con HTA resistente, correspondiendo en $10 \%$ a causas secundarias y 17\% a mayores de 60 años. Es digna de subrayar la mayor frecuencia aparente de hiperaldosteronismo primario en la HTA resistente, especialmente si se investiga midiendo la relación entre la concentración de aldosterona y la actividad de renina en plasma (Aldo/ARP) ${ }^{41}$. Aun cuando no se cumplan los criterios diagnósticos estrictos de hiperaldosteronismo primario, la concentración inapropiadamente elevada de aldosterona en sangre para la cantidad de sal ingerida o la magnitud de la volemia, puede contribuir a la patogénesis de la HTA resistente. El mineralocorticoide produce HTA no sólo por retención de sodio con la consecuente hipervolemia, sino por sus efectos vasoconstrictores directos y por alteración de la complacencia vascular ${ }^{42}$. Se han identificado receptores de aldosterona en corazón, endotelio y músculo liso arterial y en sistema nervioso central $^{43}$, lo que ha sugerido que sus efectos deletéreos en esos órganos blanco podrían ser independientes de los cambios que produce en la función renal y la PA. La aldosterona difunde directamente a través de la membrana plasmática y se acopla a su receptor inactivo citoplásmico. A continuación, ese receptor se disocia de un complejo multiproteico que, a su vez, media la translocación del receptor a través de los poros nucleares hacia la cromatina. Ya en el núcleo, el receptor activado actúa como un factor de trascripción que modula la expresión de diversas proteínas, incluyendo a la quinasa-1 inducible por glucorticoides $(\mathrm{sgk}-1)^{44}$. Esta dispara una cascada de eventos cuyo resultado es el aumento de la absorción de sodio y agua a través del canal epitelial de sodio del túbulo colector en el nefrón distal $^{45} \mathrm{e}$, indirectamente, el incremento de la excreción de potasio. Como consecuencias, aumentan la volemia y la PA. Se ha demostrado la síntesis de aldosterona fuera de las suprarrenales,

\section{Tabla 3. Indicadores clínicos de una posible causa de H TA secundaria}

1. Inicio antes de los 20 años de edad o después de los 50

2. Enfermedad renal crónica

3. Detección por estudios de imágenes de riñones "pequeños"

4. Soplo sisto-diastólico en epigastrio o flancos (estenosis de la arteria renal)

5. Palpación de ambos riñones aumentados de tamaño (poliquistosis renal)

6. Estrías purpúricas, cara de "luna llena", "jiba de búfalo" (enfermedad de Cushing)

7. Crisis hipertensiva paroxística, palpitaciones, cefalea, precordialgia, sudoración, temblor (feocromocitoma)

8. Estigmas cutáneos de neurofibromatosis de von Recklinghausen (feocromocitoma)

9. Hipokalemia no atribuible a diuréticos, astenia, poliuria, polidipsia, calambres y debilidad muscular, parestesias (hiperaldosteronismo primario)

10. PA en miembros inferiores menores a las de brazos; disminución y retardo de los pulsos en miembros inferiores (coartación de aorta)

11. HTA moderada a severa con asimetría del tamaño renal

12. Alteraciones del sueño, ronquidos, somnolencia diurna, obesidad central (apnea del sueño)

13. Daño de órgano blanco

14. HTA acelerada o maligna (retinopatía grado III-IV)

15. Súbito agravamiento de la HTA a cualquier edad

16. HTA con elevación reciente e inexplicable de la creatininemia al administrar un IECA (HTA por estenosis de arterias renales)

17. HTA resistente al tratamiento 
comprobándose que el músculo liso vascular expresa no sólo receptores mineralocorticoides, sino también la enzima 11ß-hidroxiesteroide dehidrogenasa, que confiere especificidad al receptor mineralocorticoide ${ }^{46}$. En la HTA resistente la aldosterona tisular podría ser aún más importante que la elevación leve de su concentración plasmática como causa de daño cardiovascular. La hormona promueve el depósito de colágeno en las arterias, reduciendo su elasticidad y llevando al remodelado. Asimismo, induce estrés oxidativo por activación de la $\mathrm{NAD}(\mathrm{P}) \mathrm{H}$ oxidasa, produciendo disfunción endotelial ${ }^{47,48}$. El bloqueo del receptor de mineralocorticoide aumenta la biodisponibilidad de óxido nítrico y mejora la función endotelial alterada, por disminución del estrés oxidativo ${ }^{49}$. También, se han demostrado rápidos efectos no genómicos de la aldosterona aumentando la resistencia de la vasculatura renal y produciendo $\mathrm{HTA}^{50}$. La hormona produce falla en la sensibilidad de los barorreflejos ${ }^{51}$.

Una patología emergente es la apnea obstructiva del sueño, que se asocia con HTA muchas veces resistente ${ }^{52-54}$. La hipoxemia e hipercapnia resultantes incrementan el tono simpático por estimulación de los quimiorreceptores centrales y periféricos. Asimismo, durante la apnea no se estimulan los receptores pulmonares de estiramiento, que normalmente regulan y suprimen la descarga simpática. Así, el tono simpático se incrementa inicialmente de noche y en forma transitoria, pero con el tiempo lo hace en forma permanente, produciendo HTA ${ }^{55}$. Además, se ha comprobado el aumento en la síntesis y liberación de endotelina- ${ }^{56}$, eritropoyetina ${ }^{57}$ y aldostero$n^{58}$. El tratamiento de la apnea obstructiva del sueño con presión positiva continua en vías aéreas (CPAP) mejora el control de la $\mathrm{PA}^{59}$ y disminuye la actividad simpática en los músculos.

\section{Manejo del PaCiente con HTA Resistente}

En la Figura 1 se resume el enfoque que sugerimos para la evaluación, terapéutica y seguimiento del paciente con esta condición. Se solicitará el

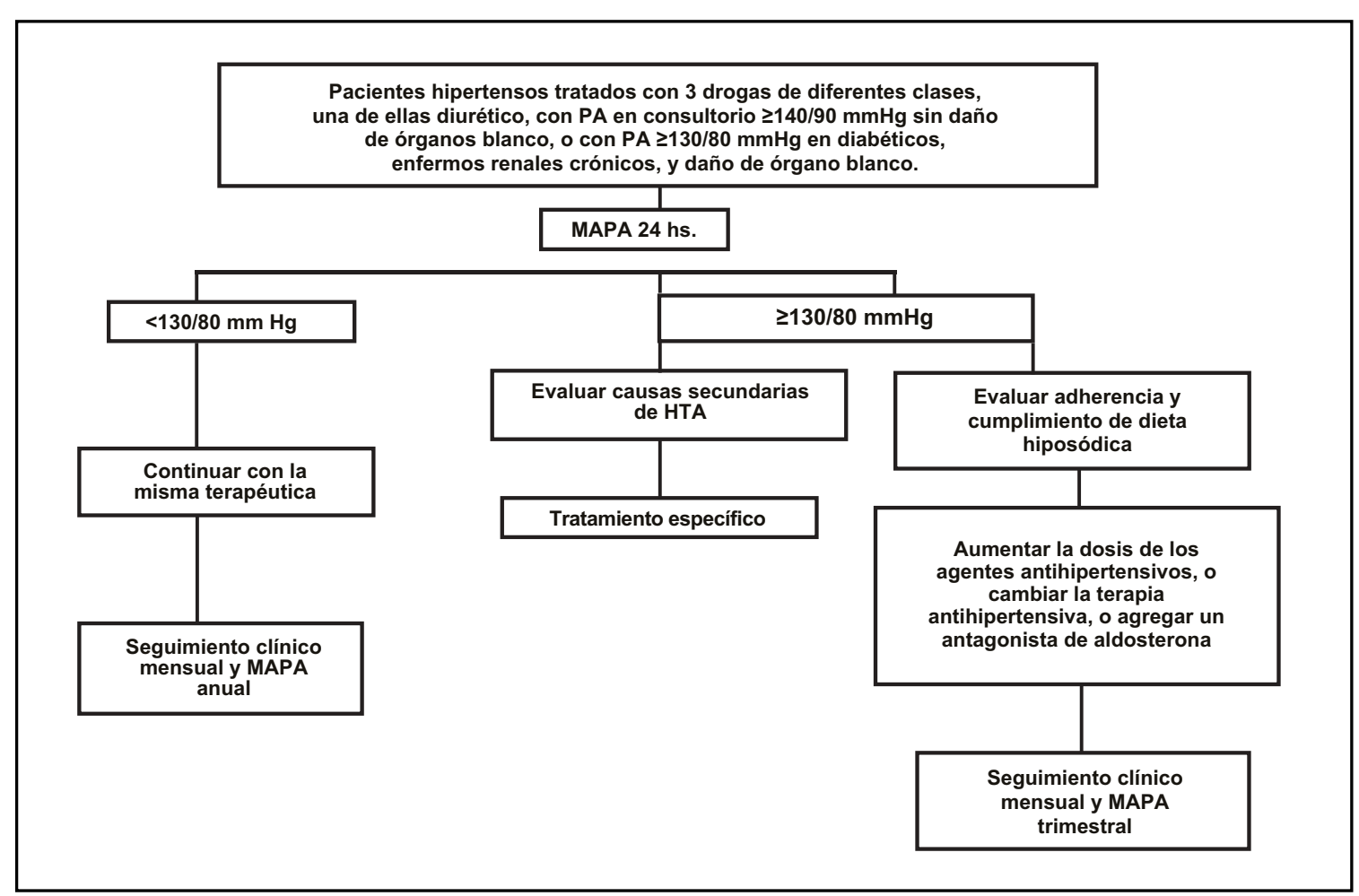

Figura 1. 
laboratorio de rutina, incluyendo hematocrito, hemoglobina, electrolitos plasmáticos, glucemia, clearance de creatinina, o por lo menos, el cálculo de la filtración glomerular por la ecuación Modification of Diet in Renal Disease (MDRD) ${ }^{60}$ o por la de Cockcroft-Gault ${ }^{61}$, perfil lipídico (colesterol total, colesterol-HDL, y trigliceridemia), orina completa con sedimento y electrocardiograma. Las posibles causas secundarias serán investigadas si el paciente tiene un cuadro clínico que así lo sugiera, siguiendo los protocolos correspondientes ${ }^{16}$. En un centro especializado de referencia se efectuará el MAPA de $24 \mathrm{~h}$, que permite distinguir entre HTA resistente y la de delantal blanco. Brown y col ${ }^{62}$ hallaron MAPA normal con descenso fisiológico de por lo menos $10 \%$ de la PA durante el sueño nocturno (comportamiento dipper) en $20 \%-30 \%$ de los pacientes con aparente HTA resistente ${ }^{63}$. Muxfeldt y cols ${ }^{13}$ observaron que $69 \%$ de sus pacientes con HTA resistente presentaron ausencia del descenso nocturno fisiológico en la PA (comportamiento non-dipper) que se asocia con mayor riesgo de complicaciones cardiovasculares, cerebrovasculares y renales ${ }^{64}$.

Se debe insistir enfáticamente a los pacientes que cumplan la dieta hiposódica, reduzcan su peso corporal en caso de sobrepeso u obesidad, realicen ejercicios aeróbicos y disminuyan la excesiva ingesta de alcohol. Las dosis de tiazidas deberán ajustarse entre $25-50 \mathrm{mg} /$ día si el clearance de creatinina es mayor de $50 \mathrm{ml} / \mathrm{min}$, pero si éste desciende por debajo de $30 \mathrm{ml} / \mathrm{min}$ serán reemplazadas por diuréticos de asa. La furosemida se administra en dosis de $20-80 \mathrm{mg} /$ día, repartida en 2-3 tomas diarias ${ }^{65}$, pues la intensa natriuresis que provoca una sola dosis dura 6-8 h y puede ser seguida por retención reactiva de sodio mediada por el sistema renina-angiotensina-aldosterona, con el consecuente inadecuado control de la PA. La torsemida, diurético de asa de acción prolonga$\mathrm{da}$, se administra en una sola dosis diaria de 2,5-5 $\mathrm{mg}$, pero tiene un mayor costo. Si el paciente continúa con cifras tensionales elevadas se deberá reevaluar el esquema terapéutico, en consulta con el especialista, administrando combinaciones de drogas de distintas familias de fármacos, teniendo en cuenta los posibles mecanismos fisiopatológicos que pudieran participar1,2,66. No se han establecido aún por estudios aleatorios apropia- dos, cuáles son los fármacos antihipertensivos indicados en tercera, cuarta y quinta línea ${ }^{2}$. Recientes estudios observacionales sugieren el beneficio de agregar agentes antialdosterona en los pacientes con HTA resistente ${ }^{48,67}$. Nishizaka y $\mathrm{Col}^{48}$, en un estudio prospectivo en pacientes con HTA resistente, agregaron dosis bajas de espironolactona $(12,5-50 \mathrm{mg} /$ día $)$ a la medicación antihipertensiva administrada. Después de un seguimiento de 6 meses, la espironolactona redujo la PAS en $25 \pm 20 \mathrm{mmHg}$ y la PAD en $12 \pm 12 \mathrm{mmHg}$, sin que se comprobaran diferencias entre los pacientes con y sin aldosteronismo primario. En el ASCOT-BPLA $^{68,69}$, de los 19.257 hipertensos incluidos, con por lo menos otro factor de riesgo adicional pero sin historia de cardiopatía coronaria, 9,2\% recibieron espironolactona como cuarta medicación en dosis promedio de $25 \mathrm{mg} /$ día, durante un periodo de 1,3 años. Se comprobó la reducción en la PA de 21,9/9,5 $\mathrm{mmHg}$. Los efectos adversos de la espironolactona consisten sobre todo en ginecomastia e impotencia sexual en hombres. Se deben a la estimulación cruzada de los receptores de andrógenos y progesterona. El fármaco está contraindicado si hay falla significativa de la función renal o hiperkalemia. En los que están recibiendo antiinflamatorios no esteroidales, aun si se prescriben dosis bajas de espironolactona (12,5 mg diarios), deben monitorearse frecuentemente la kalemia y la función renal ${ }^{70}$. Una alternativa a la espironolactona es la eplerenona, bloqueante del acoplamiento de la aldosterona al receptor mineralocorticoide, que no produce efectos hormonales adversos. Se administra en unidosis de $50 \mathrm{mg} /$ día, debiendo reducirse a $25 \mathrm{mg} /$ día si el paciente recibe medicamentos que comparten la misma vía metabólica de la CYP3A4 (konazoles, eritromicina, verapamil, saquinavir). Weinberger y cols ${ }^{71}$ en hipertensos hiporreninémicos, y White y cols ${ }^{54}$, en ancianos con HTA sistólica de difícil manejo o con HTA resistente, hallaron que la eplerenona tuvo mayor eficacia que losartan y similar efectividad que la amlodipi$\mathrm{na}^{72}$. Epstein y cols ${ }^{73}$ observaron que la eplerenona redujo la microalbuminuria en un rango mayor que el logrado solamente con la inhibición de la enzima convertidora ${ }^{73}$. Su administración está contraindicada si el clearance de creatinina es $<30$ $\mathrm{ml} / \mathrm{min}$. Es de fundamental importancia tener 
presente que los antialdosterónicos pueden provocar hiperkalemia, peligroso efecto adverso que aparece con mayor incidencia cuando se combinan con IECAs, BRAs o AINEs. Otros agentes antihipertensivos que pueden ser de utilidad en la HTA resistente son los vasodilatadores directos, como la hidralazina y el minoxidil ${ }^{74}$. Esas drogas son indicadas en cuarta o quinta línea y, en el caso de utilizárselos habrá que ajustar la dosis prescrita del diurético para prevenir la retención hidrosalina.

Si a pesar de todas esas medidas no se logra un adecuado control de la PA y hay evidencias de daño de órganos nobles, puede considerarse la selección del tratamiento basado en estudios he-

\section{REFERENCIAS}

1. Chobanian AV, Bakris GL, Black HR, Cushman WC, Green LA, Izzo JL Jr. The Seventh Report of the Joint National Committee on the Detection, Evaluation and Treatment of High Blood Pressure: the JNC VII report. JAMA 2003; 289: 2560-72.

2. Mancia G, De Backer G, Dominiczak A, Cifkova R, Fagard R, Germano G ET AL. 2007 Guidelines for the Management of Arterial Hipertension. The Task Force for the management of arterial hypertension of the European Society of Hypertension (ESH) and of the European Society of Cardiology. European Heart Journal 2007; Advance Access June 11, 2007. doi:10.1093/eurheartj/ehm236.

3. allhat Collaborative Research Group. Major outcomes in high-risk hypertensive patients randomized to angiotensin-converting enzyme inhibitor or calcium channel blocker vs diuretic: the Antihypertensive and Lipid-Lowering Treatment to Prevent Heart Attack Trial (ALLHAT). JAMA 2002; 288: 1981-97.

4. Hyman DJ, Pavlik VN. Characteristic of patients with uncontrolled hypertension in the United States. N Engl J Med 2001; 345: 479-86.

5. Taler SJ. Treatment of resistant hypertension. Curr Hypertens Rep 2005; 7: 323-9. modinámicos no invasivos como la bioimpedancia torácica $^{75}$. Se halla aún en etapa de investigación el beneficio que brindan enfoques no farmacológicos, como el equipo Rheos (CVRx, Maple Grove, MINN, USA) que estimula los barorreceptores carotí$\operatorname{deos}^{76}$. La cuidadosa evaluación de la adherencia a la medicación constituye un paso fundamental en el manejo de la HTA resistente; que puede mejorarse sustancialmente por el seguimiento telefónico o por correo electrónico, con la participación de enfermeras y otros miembros del equipo de salud, recordando a los pacientes la necesidad de cumplir adecuadamente la prescripción e interrogándoles sobre la posible presentación de potenciales efectos farmacológicos adversos.
6. Beilin LJ, Bulpitt CJ, Coles EC, Dollery CT, Gear JS, HARPER G ET al. Long-term antihypertensive drug treatment and blood pressure control in three hospital hypertension clinics. Br Heart J 1980; 43 : 74-9.

7. Anderson O, Berglund G, Hansson L, Sannerstedt R, Sivertsson R, Wikstrand J, Wilhelsem L. Organization and efficiency of an out-patient hypertension clinic. Acta Med Scand 1978; 203: 391-8.

8. Davis BR, Cutler JA, Gordon DJ, Furberg CD, Wright JT JR, Cushman WC et al. Rationale and design for the Antihypertensive and Lipid Lowering Treatment to Prevent Heart Attack Trial (ALLHAT). ALLHAT Research Group. Am J Hypertens 1996; 9: 342-60.

9. Epstein M. Resistant Hypertension: Prevalence and Evolving Concepts. J Clin Hypertens 2007; 9: 2-6.

10. Black HR, Elliott WJ, Grandits G, Grambsch P, LuCEnTE T, White WB. CONVINCE Research Group. Principal results of the Controlled onset Verapamil Investigation of Cardiovascular End Points (CONVINCE) trial. JAMA 2003; 289: 2073-82.

11. Pickering TG, James GD, Boddie C, Harshfield GA, Blank S, Laragh JH. How common is white coat hypertension? JAMA 1988; 259: 225-9.

12. Garg JP, Elliott WJ, Folker A, Izhar M, Black HR. Resistant hypertension revisited. A Comparison of 
two University-Based Cohorts. Am J Hypertens 2005; 18: 619-26.

13. Muxfeldt ES, Bloch KV, da Rocha Nogueira A, SALLES GF. True resistant hypertension: it is possible to be recognized in the office? Am J Hypertens 2005; 18: 1534-40.

14. Messerli FH, Ventura HO, Amodeo C. Osler's maneuver and pseudohypertension. N Engl J Med 1985; 312: 1548-51.

15. Wright JC, LoOney SW. Prevalence of positive Osler's maneuver in 3387 persons screened for the Systolic Hypertension in the Elderly Program (SHEP). J Hum Hypertens 1997; 11: 285-9.

16. Kaplan NM. Clinical Hypertension. $9^{\text {th }}$ Ed. Lippincott Williams \& Wilkins. 2006, pp. 280.

17. Werlemann BC, Offers E, Kolloch R. Compliance problems in therapy resistant hypertension. Herz 2004; 29: 271-5.

18. Yakovlevitch M, Black HR. Resistant hypertension in a tertiary care clinic. Arch Int Med 1991; 151 : 1786-92.

19. Amar J, Chamontin B, Genes N, Cantet C, Salvador $\mathrm{M}$, Cambou JP. Why is hypertension so frequently uncontrolled in secondary prevention? J Hypertens 2003; 21: 1199-205.

20. Berlowitz DR, Ash AS, Hickey EC, Friedman RH, Glickman M, Kader B. Inadequate management of blood pressure in a hypertensive population. $\mathrm{N}$ Engl J Med 1998; 339: 1957-63.

21. Sowers JR, White WB, Pitt B, Whelton A, Simon LS, Winer N ET AL. The effects of cyclooxygenase-2 inhibitors and nonsteroidal anti-inflammatory therapy on 24-hour blood pressure in patients with hypertension, osteoarthritis and type 2 diabetes mellitus. Arch Intern Med 2005; 165: 161-8.

22. Zanchetti A, Hansson L, Leonetti G, Rahn KH, Ruilope L, Warnold I ET AL. Low-dose aspirin does not interfere with the blood pressure-lowering effects of antihypertensive therapy. J Hypertens 2002; 20: 1015-22.

23. Davies SJC, Ghahramani P, Jackson PR. Panic disorder, anxiety and depression in resistant hypertension -a case- control study. J Hypertension 1997; 15: $1077-82$

24. Kaplan NM. Anxiety-induced hyperventilation. A common cause of symptoms in patients with hypertension. Arch Intern Med 1997; 157: 945-8.

25. Davies SJ, Jackson PR, Ramsay LE, Ghaharamani P. Drug intolerance due to nonspecific adverse effects related to psychiatric morbidity in hypertensive patients. Arch Intern Med 2003; 163: 592600.

26. Singer GM, Izhar M, Black HR. Goal-oriented hypertension management: translating clinical trials to practice. Hypertension 2002; 40: 464-9.

27. Henningsen NC, Ohlsson O, Mattiasson I, Trell E, Kristensson H, Hood B. Hypertension, levels of gamma-glutamyltranspeptidase and degree of blood pressure control in middle-aged males. Acta Med Scand 1980; 207: 245-51.

28. Gordon T, Kannel WB. Drinking and its relation to smoking, blood pressure, blood lipids and uric acid. Arch Intern Med 1983; 143: 1366-74.

29. Rakic V, Puddey IB, Burke V, Dimmitt SB, Beilin LJ. Influence of pattern of alcohol intake on blood pressure in regular drinkers: A controlled trial. J Hypertens 1998; 16: 165-74.

30. Greenberg G, Thompson S, Brennan P. The relationship between smoking and the response to antihypertensive treatment in mild hypertension in the Medical Research Council's Trial of Treatment. Intern J Epidemiol 1987; 16: 25-30.

31. Morris RC Jr, Sebastian A, Forman A, Tanaka M, Schmidlin O. Normotensive SALT sensitivity: effects of race, and dietary potassium. Hypertension 1999; 33: 18-23.

32. COWley AW JR, Roman RJ. The role of the kidney in hypertension. JAMA 1996; 275: 1581-9.

33. DAhl LK, Heine M. Primary role of renal homografts in setting chronic blood pressure levels in rats. Circ Res 1975; 36: 692-6.

34. Fox U, Bianchi G. The primary role of the kidney in causing blood pressure difference between the Milan hypertensive strain (MHS) and normotensive rats. Clin Exp Pharmacol Physiol 1976; 3: Suppl 3: 71-4.

35. Curtis JJ, Luke RG, Dustan HP et al. Remission of essential hypertension after renal transplantation. N Engl J Med 1983; 309: 1009-15.

36. Johnson RJ, Herrera-Acosta J, Schreiner GF, RodríGuEz-Iturbe B. Subtle acquired renal injury as a mechanism of salt-sensitivity hypertension. N Engl J Med 2002; 346: 913-23.

37. Bramlage P, Pittrow D, Wittchen HU, Kirch W, Boehler S, Lehnert H et AL. Hypertension in overweight and obese primary care patients is highly prevalent and poorly controlled. Am J Hypertens 2004; 17: 904-10. 
38. Van Jaarsveld BC, Krijnen P, Derkx FH, Deinum J, Woittiez AJ, Postma CT et AL. Resistance to antihypertensive medication as predictor of renal artery stenosis: comparison of two drug regimens. J Hum Hypertens 2001; 15: 669-76.

39. Wofford MR, King DS, Wyatt SB, Jones DW. Secondary hypertension: detection and management for the primary care provider. J Clin Hypertens (Greenwich) 2000; 2: 124-31.

40. Anderson GH Jr, Blakeman N, Streeten DH. The effect of age on prevalence of secondary forms of hypertension in 4429 consecutively referred patients. J Hypertens 1994; 12: 609-15.

41. Mosso L, Carvajal C, González A, Barraza A, Avila F, Montero J et al. Primary aldosteronism and hypertensive disease. Hypertension 2003; 42: 161-5.

42. Rocha R, Stier CT. Pathophysiological effects of aldosterone in cardiovascular tissues. Trends Endocrinol Metab 2001; 12: 308-14.

43. Rocha R, Rudolph AE, Friedrich GE. Aldosterone induces a vascular inflammatory phenotype in the rat heart. Am J Physiol Heart Circ Physiol 2002; 283: H1802-H1810.

44. Lombes M, OBdin ME, Gasc JM. Immunohistochemical and biochemical evidence for a cardiovascular mineralocorticoid receptor. Circ Res 1992; 71: 503-10.

45. Canessa CM, Schild L, Buell G, Thorens B, Gautschi I, Horisberge J-D, Dossier BC. Amiloride-sensitive epithelial $\mathrm{Na}^{+}$channel is made of three homologous subunits. Nature 1994: 367: 463-7.

46. TAkeda Y. Vascular synthesis of aldosterone; role in hypertensión. Mol Cell Endocrinol 2004; 217 : 75-9.

47. Schiffrin EL. Effects of aldosterone on the vasculature. Hipertension 2006; 47: 312-8.

48. Nishizaka M, Zaman MA, Calhoun D. Efficacy of low dose spironolactone in subjects with resistant hypertension. Am J Hypertens 2003; 16: 925-30.

49. Bauersachs J, Heck M, Fraccarollo D. Addition of spironolactone to angiotensin-converting enzyme inhibition in heart failure improves endothelial vasomotor dysfunction: role of vascular superoxide anion formation and endothelial nitric oxide synthase expression. J Am Coll Cardiol 2002; 39: 351-8.

50. Losel R, Feuring M, Wehling M. Non-genomic aldosterone action: from the cell membrane to human physiology. J Steroid Biochem Mol Biol 2002; 83: 167-71.

51. Schmidt BM, Horisberger K, Feuring M. Aldosterone blunts human baroreflex sensitivity by a nongenomic mechanism. Exp Clin Endocrinol Diabetes 2005; 113: 252-6.

52. Logan AG, Perlikowski SM, Mente A. High prevalence of unrecognized sleep apnea in drugresistant hypertension. J Hypertens 2001; 19: 2271-7.

53. Lavie P, Hoffstein V. Sleep apnea syndrome: a possible contributing factor to resistant hypertension. Sleep 2001; 24: 721-5.

54. Wolk R, Shamsuzzaman AS, Somers VK. Obesity, sleep apnea and hypertension. Hypertension 2003; 42: 1067-74.

55. Dimsdale JE, Coy T, Ziegler MG, Ancoli-Israel S, Clausen J. The effect of sleep apnea on plasma and urinary catecholamines. Sleep 1995; 18: 377-81.

56. Phillips BG, Narkiewicz K, Pesek CA, Haynes WG, Dyken ME, Somers VK. Effects of obstructive sleep apnea on endothelin-1 and blood pressure. J Hypertens 1999; 17: 61-6.

57. Winnicki M, Shamsuzzaman A, Lanfranchi P, Accurso V, Olson E, Davison D et al. Erythropoietin and obstructive sleep apnea. Am J Hypertens 2004; 17: 783-6.

58. Calhoun DA, NishizaKa MK, Zaman MA. Aldosterone excretion among subjects with resistant hypertension and symptoms of sleep apnea. Chest 2004; 125: 112-7.

59. Goodfriend TL, Calhoun DA. Resistant hypertension, obesity, sleep apnea, and aldosterone. Hypertension 2004; 43: 518-24.

60. Levey AS, Bosch JP, Lewis JB, Greene T, Rogers N, Roth D. A more accurate method to estimate glomerular filtration rate from serum creatinine: a new prediction equation. Modification of diet in Renal Disease Study Group. Ann Intern Med 1999; 130: 461-70.

61. Stevens L, Coresh J, Greene T, Levey AS. Assessing kidney function-measured and estimated glomerular filtration rate. N Engl J Med 2006; 354 : 2473-83.

62. Brown MA, Buddle ML, Martin A. Is resistant hypertension really resistant? Am J Hypertens 2001; 14: 1263-9.

63. O'Brien E, Sheridan J, O'Malley K. Dippers and non-dippers. Lancet 1988; 2: 397. 
64. Verdecchia P, Porcelatti C. Circadian blood pressure changes and left ventricular hypertrophy in essential hypertension. Circulation 1990; 81: 52836.

65. Finnerty FA, MaXwell MH, Lunn J, Moser M. Longterm effects of furosemide and hydrochlorothiazide in patients with essential hypertension: a two-year comparison of efficacy and safety. Angiology 1977; 28: 125-33.

66. Moser M, Setaro JF. Resistant or difficult to control hypertension. N Engl J Med 2006; 355: 385-92.

67. Ouzan J, Perault C, Lincoff M, Carre E, Mertes M. The role of spironolactone in the treatment of patients with refractory hypertension. Am J Hypertens 2002; 15 N: 333-9.

68. Sever PS, Dahlof B, Poulter NR, Wedel H, Beevers G, Caufield M et al., for the ASCOT investigators. Rationale, design, methods and baseline demography of the Anglo-Scandinavian Cardiac Outcomes Trial. J Hypertens 2004; 18: 139-85.

69. Chapman N, Dobson J, Wilson S, Dahlöf, Sever PS, Wedel H ET AL. Effect of Spironolactone on blood pressure in subjects with resistant hypertension. Hypertension 2007; 49: 839-45.
70. Calhoun DA. Use of Aldosterone antagonists in resistant hypertension. Progress in Cardiovascular Diseases 2006; 48: 387-96.

71. Weinberger MH, White WB, Ruilope LM. Effects of eplerenone versus losartan in patients with low renin hypertension. Am Heart J 2005; 150: 426-33.

72. White WB, Duprez D, Hillaire R. Effects of the selective aldosterone blocker eplerenone versus the calcium antagonist amlodipine in systolic hypertension. Hypertension 2003; 41: 1021-6.

73. Epstein M, Wildiams GH, Weinberger M. Selective aldosterone blockade with eplerenone reduces albuminuria in patients with type 2 diabetes. Clin J Am Soc Nephrol 2006; 1: 940-51.

74. SICA DA. Minoxidil: an underused vasodilator for resistant or severe hypertension. J Clin Hypertens (Greenwich) 2004; 6: 283-7.

75. Taler SI, Textor SC, Augustine JE. Resistant hypertension. Comparing hemodynamic management to specialist care. Hypertension 2002; 39: 982-8.

76. Lohmeier TE, Dwyer TM, Irwin ED, Rossing MA, KIEVAL RS. Influence of prolonged baroreflex activation on arterial pressure in angiotensin hypertension. Hypertension 2005; 46: 1194-200. 